\title{
Spätglaziale äolische Ablagerungen in einigen Mittelgebirgen Hessens ${ }^{1}$ )
}

\author{
Von ERnst Schönhals, Wiesbaden
}

Mit 4 Abb. im Text

$\mathrm{Zusammenfassung.} \mathrm{Aus} \mathrm{den} \mathrm{Hochlagen} \mathrm{von} \mathrm{Vogelsberg,} \mathrm{Taunus} \mathrm{und} \mathrm{Rhön} \mathrm{werden}$ äolische Ablagerungen beschrieben, die - wie mit großer Wahrscheinlichkeit angenommen werden darf - durch Westwinde angeweht worden sind. Es handelt sich nicht um ferntransportiertes Material, sondern jeweils um eine Lokalfazies, was aus der Abhängigkeit der Körnung und des Mineralgehaltes von den Gesteinen der unmittelbaren Umgebung hervorgeht.

Auf Grund von Besonderheiten des Substrates, des an mehreren Stellen des Hohen Vogelsberges aufgefundenen Humushorizontes und der von der Morphologie abhängigen Verbreitung werden die geringmächtigen Deckschichten dem Spätglazial, und zwar der Jüngeren Tundrenzeit, zugewiesen. Das Klima dieses Zeitabschnittes hatte also in den Hochlagen der genannten Gebirge einen periglazialen Charakter.

Abstract. Aeolian deposits, which have been found in the high parts of Vogelsberg, Taunus and Rhön (Western Germany) were transported probably by western winds. It is a local material, which didn't come from afer. The age of this aeolian cover is Late-Glacial, presumably belonging to the Younger Dryas time. In the higher parts of the above-mentioned mountains, the climate of the Younger Dryas time was periglacial.

\section{In ha $1 \mathrm{t}$}

Einleitung

1. Vogelsberg

a) Allgemeines

b) Die periglazialen Ablagerungen des Oberwaldes

2. Taunus

a) Allgemeines

b) Die Deckschichten

3. Rhön

4. Entstehung und Alter der Deckschichten.

\section{Einleitung}

Von allen minerogenen Bildungen des gletscherfreien Gebietes Mitteleuropas haben die weit verbreiteten äolischen Sedimente wohl mit die größte Bedeutung. Da ihre Entstehung nur bei einem ganz bestimmten Klima möglich war, geben sie uns wichtige Anhaltspunkte für das Klima jener Zeitabschnitte, insbesondere für die vorherrschende Windrichtung und die Niederschlagshöhe.

Bei der Gliederung und Datierung periglazialer Ablagerungen spielen daher der in verschiedener Fazies vorkommende Löß und Flugsand eine besondere Rolle, zumal beide an ihrer charakteristischen Korngrößenzusammensetzung leicht zu erkennen sind. Eine stratigraphische Unterteilung vieler wichtiger Pleistozänprofile wäre ohne die Einschaltung äolischer Schichten nicht oder nur schwer möglich gewesen. Im letzten Jahrzehnt haben hierbei auch die fossilen Böden mehr und mehr an Bedeutung gewonnen.

1) Vortrag, gehalten anläßlich der Hauptversammlung der Deutschen Quartärvereinigung in Laufen a. d. Salzach am 4. 9. 1955. 
Die mächtigsten äolischen Deckschichten bestehen aus Löß. der - wie bereits vor 31/2 Jahrzehnten nachgewiesen wurde (SOERgel 1919) - während des Maximums der einzelnen Vereisungen, also unter einem trocken-kalten Klima, angeweht wurde. Der Flugsand mit seinen in zahlreichen Gebieten nachweisbaren faziellen Übergängen zum Löß tritt demgegenüber flächen- und mengenmäßig stark zurück. Nur einige Gebiete, so vor allem die Landschaften nördlich der Lößgrenze (z. B. Nordwest-Deutschland und Holland), sowie die Randbezirke vieler Flüsse des gletscherfreien Raumes (Rhein, Elbe in Böhmen, Gebiet zwischen Donau und Theiß) weisen auch diese gröberen Ablagerungen in größerer Verbreitung auf (E. Schönhals 1953). Der weitaus größte Teil dieser sandigen Decksedimente gehört bereits dem Spätglazial an. Außerhalb der erwähnten Verbreitungsgebiete, also vor allem in den Mittelgebirgen, sind bisher spätglaziale äolische Ablagerungen nicht bekannt geworden. Verfasser glaubt nun, im Vogelsberg, im Taunus und in der Rhön solche spätglazialen Sedimente gefunden zu haben. Es seien daher im folgenden zunächst die Beobachtungen und Untersuchungsergebnisse mitgeteilt. Anschließend wird dann das Alter der Deckschichten näher erörtert werden.

\section{Vogelsberg}

a) Allgemeines

Während in den flacheren Randgebieten des Gebirges (Wetterau, Vorderer Vogelsberg, Ebsdorfer Grund, Umgebung von Alsfeld und Romrod) der würmzeitliche Löß noch größere Flächen bedeckt, nimmt seine Verbreitung mit zunehmender Höhe und Steilheit derHänge rasch ab. Die letzten größeren Decken liegen zwischen 300 und $350 \mathrm{~m}$; dann wird der Lößlehm mehr und mehr von Solifluktionsmassen abgelöst. Nur in den meist kleinen Plateaulagen, die jedoch in der Westhälfte des Gebirges nicht sehr zahlreich sind, ist noch Lößlehm verbreitet. Eine größere, geschlossene Lehmdecke findet sich erst wieder im höchsten, über $700 \mathrm{~m}$ hohen Teil des Gebirges, dem sog. O b e r w a $1 \mathrm{~d}$. SCHOTTLER (1931) hat bei der geologischen Kartierung des Blattes Ulrichstein den Lehm als „kalkfreien Löß“ bezeichnet. Er weist bereits darauf hin, daß es sich bei dem Löß um eine „in verschiedenen Abschnitten der Diluvialzeit“ entstandene Ablagerung handelt, die als die "auffallendste des Hohen Vogelsberges“ bezeichnet wird. Schottler unterschied einen primären, steinfreien Löß der ebenen Flächen und einen darunter folgenden steinigen Lehm. Auf der geologischen Karte konnten die beiden Ablagerungen jedoch nicht getrennt dargestellt werden. Auffallend ist nun, daß aus der oberen dünnen Lößlehmdecke, die als ein extrem basenarmes Substrat bezeichnet werden muß (S-Werte unter $2 \mathrm{mval} / 100 \mathrm{~g}$ Boden), bei einer mittleren jährlichen Niederschlagshöhe von über $1100 \mathrm{~mm}$ und einer mittleren Jahrestemperatur von $5,5-6^{\circ} \mathrm{C}$ kein podsolierter und staunasser Boden (podsolierter Pseudogley) hervorgegangen ist, sondern ein Boden, der im gesamten Profil eine leuchtendbraune Farbe und eine optimale Lockerheit besitzt. Nennenswerte Verlagerungen kolloidaler Stoffe sind am Bodenprofil nicht zu beobachten und auch analytisch nicht nachzuweisen. Es sind daher auch keine deutlich unterscheidbaren Bodenhorizonte ausgebildet. Auf Grund des Profilaufbaues und der analytisch ermittelten Eigenschaften gehört der Boden zur Klasse der Braunerden; er wird wegen seiner besonders ins Auge fallenden Eigenschaft als "Lockerbraunerde“ bezeichnet. Demgegenüber gliedern sich die aus dem basenreichen Löß der niederschlagsärmeren (ca. 700 bis $800 \mathrm{~mm}$ ) Randgebiete hervorgegangenen Böden in einen deutlichen, bis zu $1 \mathrm{~m}$ betragenden Auswaschungshorizont (Ae) und einen verdichteten, staunassen Einwaschungshorizont $(\mathrm{gB})$. Ein weiterer Hauptunterschied zwischen den genannten Bodenbildungen besteht in dem Gefüge: Die Böden des Oberwaldes sind trotz extremer Basenarmut optimal locker, sehr hohlraumreich und stark durchlässig. Die Lößböden der Randzonen dagegen haben in dem basenärmeren Auswaschungshorizont Einzelkornstruktur und neigen daher zur Dichtschlämmung und Verkrustung. Der bei Austrocknung in Säulen 
abgesonderte und in vieleckige Bruchkörper zerfallende Anreicherungshorizont ist dicht und je nach dem Grad der Staunässe, der im wesentlichen von der Geländeform abhängt, \pm stark fleckig (pseudovergleyt). Eine Erklärung der Bodenunterschiede zwischen dem niedrigeren Vorderen Vogelsberg und dem über $700 \mathrm{~m}$ hohen Oberwald bereitete seither Schwierigkeiten. Klima, Vegetation und Geländeform reichen zur Erklärung nicht aus. Verfasser ist nun der Ansicht, daß die Diskrepanz in der Bodenentwicklung auf das jüngere geologische Alter der Ablagerungen im Oberwald zurückzuführen ist.

b) Die periglazialen Ablagerungendes Oberwaldes

Die vulkanischen Gesteine (verschiedenartige Basalte, in geringer Verbreitung Tuff und an einer Stelle Phonolith) sind mit Ausnahme der steilen Hänge und Kuppen von Solifluktionsschutt überzogen. Seine Mächtigkeit erreicht mehrere Meter. Er besteht aus
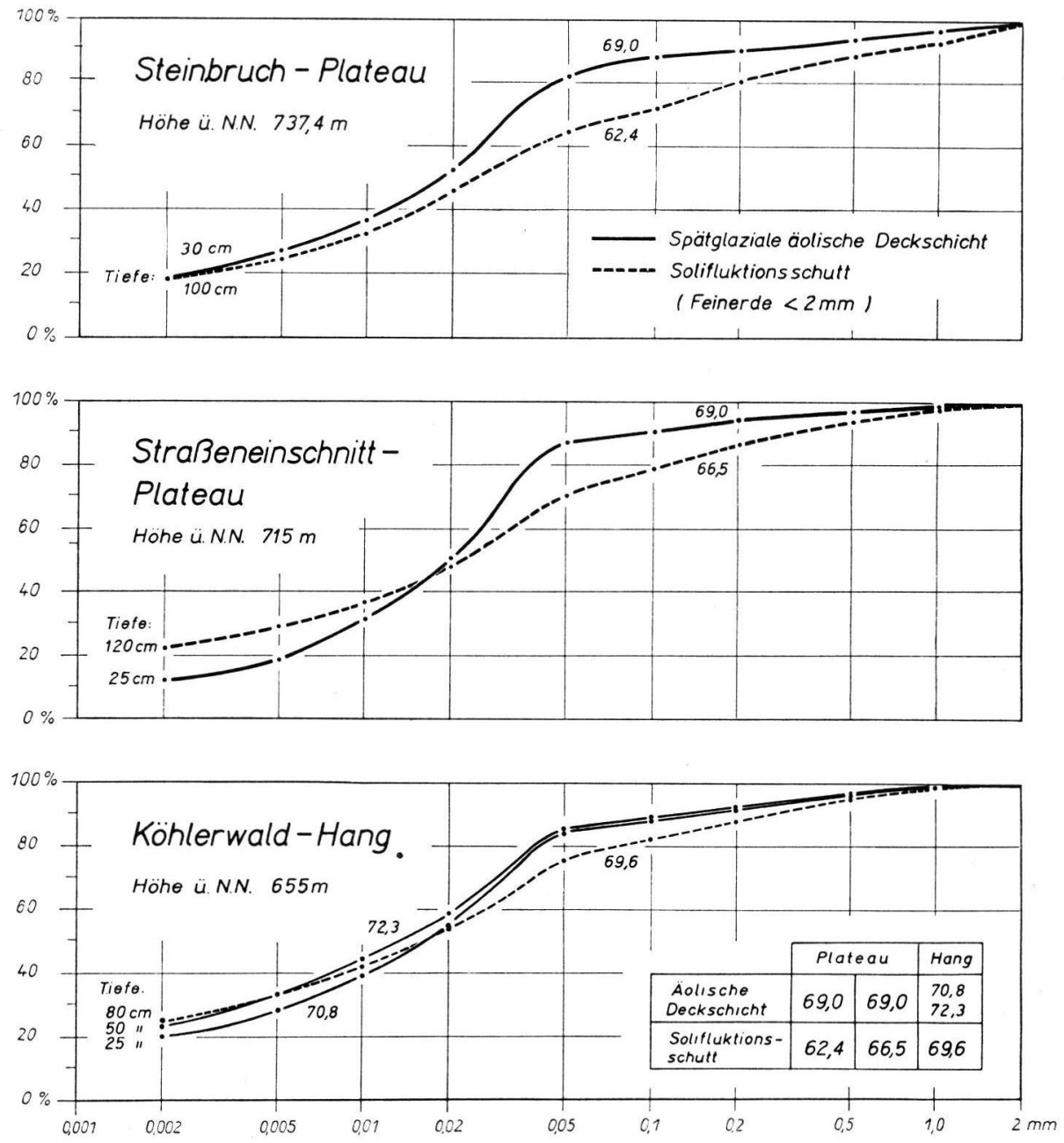

Abb. 1. Kornverteilungskurven des Solifluktionsschuttes und der äolischen Deckschicht des Hohen Vogelsberges (Oberwald). Die Feinheit der einzelnen Proben ist aus den Kennzahlen zu ersehen. Der Feinheitsgrad schwankt zwischen 10 und 100 (größte Feinheit). 
fahlbraunem Lößlehm und Basaltmaterial verschiedener Größe (Grus bis Block). Der Skelettanteil wechselt. Das Gefüge des Lehms ist dicht und hohlraumarm.

Der Basaltschutt wird im Oberwald durch einen geringmächtigen, fast skelettfreien Lehm der Beobachtung weitgehend entzogen; nur an den Hängen des Oberwaldplateaus tritt er zutage. Im Gegensatz zum liegenden Solifluktionsschutt ist die bis $\mathrm{zu} 80 \mathrm{~cm}$ mächtig werdende lehmige Deckschicht locker, leuchtendbraun und stark durchwurzelt.

Um näheren Aufschluß über den Körnungsaufbau zu erhalten, wurden Proben aus dem dichten Solifluktionsschutt und der lockeren Deckschicht untersucht. Die Ergebnisse der an zwei Stellen des Oberwaldplateaus und an einer Stelle des Oberwaldhanges entnommenen Proben (Schutt und Deckschicht) sind aus Abb. 1 und Tabelle $1 \mathrm{zu}$ ersehen. Bei allen Proben überwiegt der Staub $(0,01-0,05 \mathrm{~mm} \phi)$; der Tongehalt ist verhältnismäßig hoch. Gröbere Bestandteile sind an der Zusammensetzung der Deckschicht nur mit wenigen Prozent beteiligt.

Die Feinerde des Schuttes und die Deckschicht bestehen also zu einem erheblichen Teil aus Lößmaterial. In der Korngrößenzusammensetzung des Schuttes und der Deckschicht besteht jedoch ein bemerkenswerter Unterschied: Die Deckschicht weist einen $7-21 \%$ betragenden höheren Staubanteil auf als der liegende Schutt. Das zeigen die graphischen Darstellungen der Korngrößenanalysen besonders deutlich; denn während die Kurven des Schuttes einen flachen Verlauf haben, steigen die Kurven der Deckschicht im Bereich der Staubfraktion steiler an (Abb. 1). Im allgemeinen verlaufen die Körnungslinien der Deckschicht über denen des Schuttes. Die Deckschicht ist also feiner als die Feinerde des Solifluktionsschuttes. Das gleiche kann auch aus den Kennzahlen des Feinheitsgrades geschlossen werden (letzte Spalte in der Tabelle 1 und Abb. 2; vgl. E. Schönhals 1955). Am stärksten sind die Feinheitsunterschiede zwischen Deckschicht und Schutt auf dem Oberwaldplateau, während sie am Hang (Profil Köhlerwald) geringer sind. Dies ist wahrscheinlich auf Verlagerungsvorgänge während der Entstehung zurückzuführen.

Aus den Untersuchungsergebnissen geht hervor, daß die Deckschicht durch die Wirkung des Windes entstanden ist. Der geringe gröbere Anteil $(>0,5 \mathrm{~mm})$ dürfte auf der Erdoberfläche verfegt worden sein. Nach einer petrographischen Untersuchung ${ }^{2}$ ) handelt es sich neben dunklen Basaltsplittern und -körnern fast ausschließlich um Quarzstaub mit einzelnen Plagioklaskristallen, die aus dem Basalt stammen. Sowohl der hohe Quarzgehalt als auch die stark vertretene Staubsandfraktion lassen den Schluß zu, daß die Deckschicht angeweht worden ist. Sehr wahrscheinlich haben wir kein weit transportiertes Substrat vor uns, sondern eine Lokalfazies, die im übrigen Vogelsberg bisher nicht nachgewiesen werden konnte.

Daß zwischen der Entstehung des liegenden Solifluktionsschuttes und der Deckschicht eine gewisse Zeitspanne gelegen hat, konnte im Jahr 1954 nachgewiesen werden. In neuen, bei standortskundlichen Untersuchungen der Forstverwaltung entstandenen Aufgrabungen im Oberwald wurde nämlich an 4 Stellen zwischen Basaltschutt und äolischer Deckschicht ein humoser Horizont von etwa $4-10 \mathrm{~cm}$ Mächtigkeit beobachtet. Leider enthält der lehmige Humushorizont - von eingewaschenen rezenten Pollen abgesehen - keine verwertbaren Pollen. Eine durch die Pollenanalyse gesicherte stratigraphische Einordnung ist daher nicht möglich ${ }^{3}$ ). Immerhin kann aus dem Humushorizont geschlossen werden, daß zwischen der Bildung des Solifluktionsschuttes und der Deckschicht während einer gewissen Zeit eine Vegetationsdecke bestanden hat.

$\left.{ }^{2}\right)$ Die Untersuchung wurde dankenswerter Weise von Herrn Regierungsgeologen Prof. Dr. H. Hentschel, Wiesbaden, durchgeführt.

$\left.{ }^{3}\right)$ Die pollenanalytische Untersuchung führte Herr Prof. Dr. Firbas, Göttingen, durch, wofür auch an dieser Stelle herzlich gedankt sei. 
Tabelle 1

Korngrößenzusammensetzung der spätglazialen äolischen Deckschichten und des würmeiszeitlichen Solifluktionsschuttes in einigen Mittelgebirgen Hessens

\begin{tabular}{|c|c|c|c|c|c|c|c|c|c|c|c|c|c|c|c|}
\hline Landschaft & Lage & $\begin{array}{l}\text { Geol. } \\
\text { Bez. }\end{array}$ & $\begin{array}{l}\text { Tiefe der } \\
\text { Probe- } \\
\text { entnahme }\end{array}$ & $\begin{array}{c}<0,002 \\
\mathrm{~mm}\end{array}$ & $\begin{array}{c}0,002- \\
0,005 \\
\mathrm{~mm}\end{array}$ & $\begin{array}{l}0,005- \\
0,01 \\
\mathrm{~mm}\end{array}$ & $\begin{array}{l}0,01- \\
0,02 \\
\mathrm{~mm}\end{array}$ & $\begin{array}{l}0,02 \\
0,05 \\
\mathrm{~mm}\end{array}$ & $\begin{array}{l}\mathbf{0 , 0 5 -} \\
\mathbf{0 , 1} \\
\mathrm{mm}\end{array}$ & $\begin{array}{l}0,1- \\
0,2 \\
\mathrm{~mm}\end{array}$ & $\begin{array}{l}0,2- \\
0,5 \\
\mathrm{~mm}\end{array}$ & $\begin{array}{l}0,5- \\
1,0 \\
\mathrm{~mm}\end{array}$ & $\begin{array}{l}1,0- \\
2,0 \\
\mathrm{~mm}\end{array}$ & $\begin{array}{l}>2,0 \\
\mathrm{~mm}\end{array}$ & $\begin{array}{l}\text { Kenn- } \\
\text { zahl }\end{array}$ \\
\hline \multirow{14}{*}{$\begin{array}{l}\text { Hoher } \\
\text { Vogelsberg } \\
\text { (Oberwald) }\end{array}$} & \multirow{4}{*}{$\begin{array}{l}\text { Plateau } \\
\text { Steinbruch } \\
\text { Höhe N. N.: } \\
737,4 \mathrm{~m}\end{array}$} & \multirow{2}{*}{ Dä } & \multirow{2}{*}{30} & & & & \multicolumn{2}{|c|}{46,0} & & \multirow[b]{2}{*}{2,4} & \multirow[b]{2}{*}{3,9} & \multirow[b]{2}{*}{2,6} & \multirow[b]{2}{*}{2,8} & \multirow[b]{2}{*}{2,0} & \multirow{2}{*}{69,0} \\
\hline & & & & 18,6 & 9,0 & 9,0 & 170 & 29,0 & 5,7 & & & & & & \\
\hline & & \multirow{2}{*}{$\mathbf{S}$} & \multirow{2}{*}{100} & & & & \multicolumn{2}{|c|}{32,0} & & \multirow[b]{2}{*}{8,9} & \multirow[b]{2}{*}{7,7} & \multirow[b]{2}{*}{3,6} & \multirow[b]{2}{*}{7,2} & \multirow{2}{*}{8,7} & \multirow{2}{*}{62,4} \\
\hline & & & & 18,1 & 6,0 & 9,5 & 13,0 & $\overrightarrow{19,0}$ & 7,0 & & & & & & \\
\hline & \multirow{4}{*}{$\begin{array}{l}\text { Plateau } \\
\text { Straßen- } \\
\text { einschnitt } \\
\text { Höhe N. N.: } \\
715 \mathrm{~m}\end{array}$} & \multirow[t]{2}{*}{ Dä } & \multirow[t]{2}{*}{$2 \bar{s}$} & & & & \multicolumn{2}{|c|}{56.0} & & & & & & & \multirow[t]{2}{*}{69,0} \\
\hline & & & & 12,3 & 6,3 & 11,6 & 19,5 & 36,5 & 4,2 & 4,2 & 2,4 & 2,2 & 0,8 & 0 & \\
\hline & & \multirow{2}{*}{$\mathrm{s}$} & \multirow{2}{*}{120} & & & & \multicolumn{2}{|c|}{34,9} & & & & & & & \multirow{2}{*}{66,5} \\
\hline & & & & 22,1 & 7,5 & 6,5 & 12,5 & 22,4 & 7,8 & 7,9 & 7,2 & 4,7 & 1,4 & 3,8 & \\
\hline & & & 25 & & & & & & & & & & & & 708 \\
\hline & О.Нап & 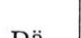 & & 21,1 & 9,0 & 9,9 & 15,5 & 29,1 & 3,3 & 4,5 & 4,7 & 2,0 & 0,9 & 6,4 & \\
\hline & Köhlerwald & $D d$ & 50 & & & & & & & & & & & & 72.3 \\
\hline & Höhe N. N.: & & & 24,6 & 9,5 & 10,4 & $\overparen{14,5}$ & 26,0 & 4,1 & 3,1 & 4,1 & 1,8 & 1,9 & 9,4 & \\
\hline & & $\mathrm{s}$ & 80 & & & & & & & & & & & & 69,6 \\
\hline & & & & 25,1 & 9,0 & 8,0 & 12,0 & 21,4 & 7,1 & 5,8 & 7,1 & 2,9 & 1,6 & 17,0 & \\
\hline & OHang & & 50 & & & & & & & & & & & & 46.4 \\
\hline Hochtaunus & Hohe Wurzel & 范 & & 2,6 & 5,1 & 4,4 & 9,5 & $\overline{18,2}$ & 4,3 & $\overparen{13,5}$ & $\overline{25,8}$ & 12,1 & 4,5 & 1,8 & \\
\hline & Höhe N. N.: & & 70 & & & & & & & & & & & & 53.8 \\
\hline & & & & 8,0 & 7,9 & 7,5 & $\overparen{12,0}$ & $\overline{13,0}$ & 4,7 & 12,9 & $\overrightarrow{25,5}$ & 5,0 & 3,5 & 3,6 & \\
\hline & & & 20 & & & & & & & & & & & & 498 \\
\hline & S-Hang & & & 2,3 & 2,1 & 4,5 & $\overparen{14,9}$ & 25,2 & 7,7 & $\overparen{11,4}$ & 20,8 & 7,4 & 3,7 & 1,7 & 75,0 \\
\hline Rhön & $\begin{array}{l}\text { der } \\
\text { Wasserkunpe }\end{array}$ & Dä & 35 & & & & & & & & & & & & 52,5 \\
\hline & Höhe N. N.: & & & 2,9 & 4,4 & 7,9 & 20,2 & 21,1 & 4,2 & 9,0 & 14,3 & 9,3 & 6,7 & 25,7 & \\
\hline & $930 \mathrm{~m}$ & & 60 & & & & & & & & & & & & 48.3 \\
\hline & & & & 2,7 & 3,7 & 6,2 & 14.9 & 19,6 & 5,1 & $\overparen{11,0}$ & $\overline{16,9}$ & 10,9 & 9,0 & 32,0 & \\
\hline
\end{tabular}




\section{Taunus}

a) Allgemeines

Der Kamm des Taunus wird bekanntlich aus unterdevonischen Quarziten und Sandsteinen aufgebaut. Im Bereich der Blätter Eltville-Heidenfahrt, Bad-Schwalbach und Wehen ist der Quarzitrücken im allgemeinen 450-580 m hoch. Nur an zwei Stellen wird die 600 m-Linie überschritten („Kalte Herberge“ 619,5 m, „Hohe Wurzel“ 613,9 m). Die Hänge des SW-NO-streichenden Gebirgszuges werden weithin von Solifluktionsschutt eingenommen, der fast immer skelettreich ist. Ein höherer Schluff- und Staubsandgehalt ist meist vorhanden. Er ist auch die Ursache der staunassen Böden, die vorwiegend an schwach geneigten Hängen und in extremer Ausbildung auf ebenen Flächen verbreitet sind.

Bei der forstlichen Standortsaufnahme der Forstämter Chausseehaus und Sonnenberg (nordwestlich von Wiesbaden) wurden nun neben podsolierten Böden auf Taunusquarzit und staunassen Böden auf Gehängeschutt braune, lehmige Böden beobachtet, die keinerlei Auswaschungserscheinungen oder Anzeichen einer Verdichtung und Vernässung aufweisen. Sie haben vielmehr die gleiche leuchtendbraune Farbe und optimale Lockerheit wie die Oberwaldböden. Dabei ist auch dieses Substrat extrem basenarm (S-Wert unter $2 \mathrm{mval} / 100 \mathrm{~g}$ Boden). Eine Erklärung für das Vorkommen dieser Böden in den niederschlagsreichen Hochlagen des südwestlichen Taunus $(750-800 \mathrm{~mm})$ konnte zunächst nicht gegeben werden. Anläßlich einer Besichtigung von Bodenprofilen im Arbeitsgebiet von Herrn Forstmeister ReInfurth ${ }^{4}$ ) wurde vom Verfasser auf die große Ähnlichkeit dieser Böden mit denen des Oberwaldes hingewiesen. Die Untersuchung eines Bodenprofils bestätigte dann auch, daß es sich ebenfalls um äolische Ablagerungen handelt.

b) Die Deckschichten

Wie aus Abb. 2 und 3 hervorgeht, treten die braunen Deckschichten in den beiden genannten Forstämtern nur auf verhältnismäßig kleinen Flächen auf. Meist haben diese eine schmale, langgestreckte Form und finden sich mitunter unmittelbar an den von mächtigerem Gehängeschutt freien steileren Hängen. So liegt ein über $2 \mathrm{~km}$ langes und z. T. über $200 \mathrm{~m}$ breites Vorkommen an dem steilen SO-Hang des 500 bis $530 \mathrm{~m}$ hohen Rückens an der "Platte“ (Abb. 2). Kleinere isolierte Vorkommen finden sich auf dem "Eichel-Berg" in 510 bis $536 \mathrm{~m}$ Höhe und am "Alten-Stein “ zwischen 460 und $490 \mathrm{~m}$. Eine $2 \mathrm{~km}$ lange und $80-250 \mathrm{~m}$ breite Decke erstreckt sich in 520 bis $580 \mathrm{~m}$ Höhe am Ostabhang der "Hohen Wurzel“ $(613,9 \mathrm{~m})$ in nordöstlicher Richtung (Abb. 3). Etwas kleinere Flächen liegen in dem Revier "Rumpelkeller" sowie nördlich der "Hohen Wurzel“ und östlich vom „Roß-Kopf“. Sehr kleine Vorkommen wurden am „Hangenstein“ und an den Hängen des „Rote-Kreuz-Kopfes“ festgestellt. - Die Mächtigkeit aller Decken ist gering; sie beträgt im allgemeinen $40-50 \mathrm{~cm}$.

Von den durchgeführten Laboratoriumsuntersuchungen ist hier nur die Korngrößenzusammensetzung von Wichtigkeit. Die Körnung der beiden am O-Hang der „Hohen Wurzel" entnommenen Proben ist aus Tabelle 1 und Abb. $4 \mathrm{zu}$ ersehen. Danach handelt es sich um einen sehr tonarmen Sand mit 25-28\% Staub $(0,01-0,05 \mathrm{~mm} \phi)$. Wie die Körnungsanalyse erkennen läßt, besitzt das Substrat zwei Körnungsmaxima, und zwar eines in der Staubsandfraktion und ein zweites in der Fraktion $0,2-0,5 \mathrm{~mm} \phi$. Die Ablagerung besteht also zum Teil aus Löß und zu einem etwas größeren Teil aus Flugsand. Die Lößkomponente dürfte aus dem Gehängeschutt der Umgebung stammen, der ebenfalls einen geringen Tongehalt (meist unter 10\%), aber einen höheren Sandgehalt

$\left.{ }^{4}\right)$ Herrn Forstmeister U! ReInfurth sei für die Uberlassung seiner standortskundlichen Aufnahmeergebnisse bestens gedankt. 


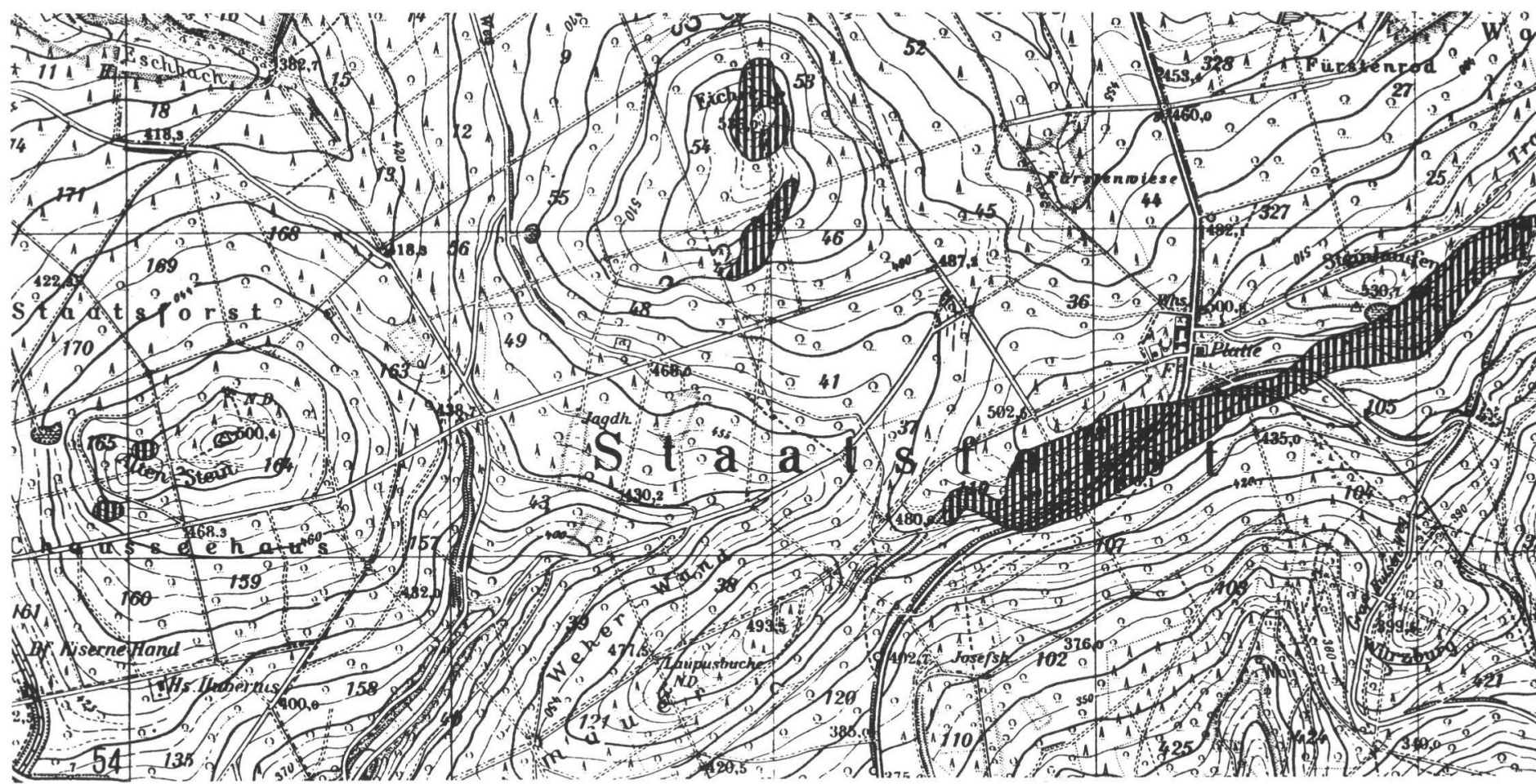

Abb. 2. Verbreitung der äolischen Deckschicht im Staatsforst Sonnenberg und im Nordostteil des Staatsforstes Chausseehaus (Maßstab $1: 25000$ ). (Topogr. Grundlage vom Hess. Landesvermessungsamt, Wiesbaden) 


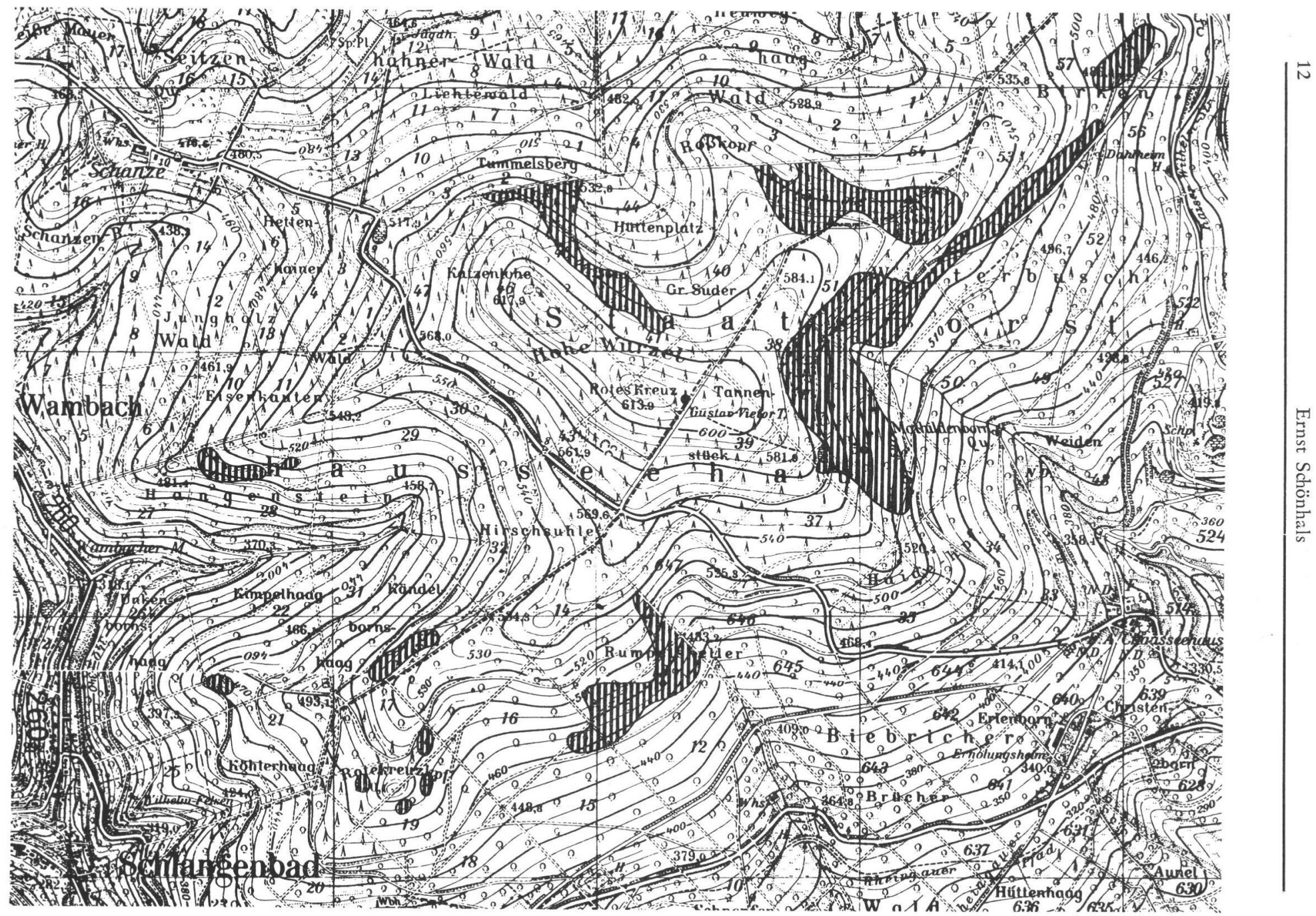

Abb. 3. Verbreitung der äolischen Deckschicht im Staatsforst Chausseehaus (Maßstab $1: 25$ 000). 
besitzt. Ein Teil des Sandes könnte aus dem Gehängeschutt herrühren. Es ist aber auch möglich, daß Sand aus dem verwitterten, in unmittelbarer Nähe anstehenden Taunusquarzit und den Sandsteinen der Hermeskeilschichten ausgeweht worden ist.

\section{Rhön}

Bisher wurden nur 3 Proben aus einem Bodenprofil am Südabhang der westlichen Wasserkuppe untersucht, wo in etwa $930 \mathrm{~m}$ über NN ebenfalls durch starke Lockerheit ausgezeichnete Böden vorkommen. Der hohe Humusgehalt verleiht den sehr basenarmen Böden eine schwarzbraune Farbe, die bis etwa $40 \mathrm{~cm}$ reicht. Wie aus den drei Körnungsanalysen hervorgeht, ist das Substrat in den gleichen Fraktionen wie das aus dem Taunus beschriebene durch zwei Maxima ausgezeichnet (Tab. 1 und Abb. 4). Allerdings überwiegt etwa mit den gleichen Anteilen wie im Oberwald der Staub; die Fraktionen $0,1-0,2$ und $0,2-0,5 \mathrm{~mm}$ sind weniger vertreten als im Taunus, besonders die Korn-
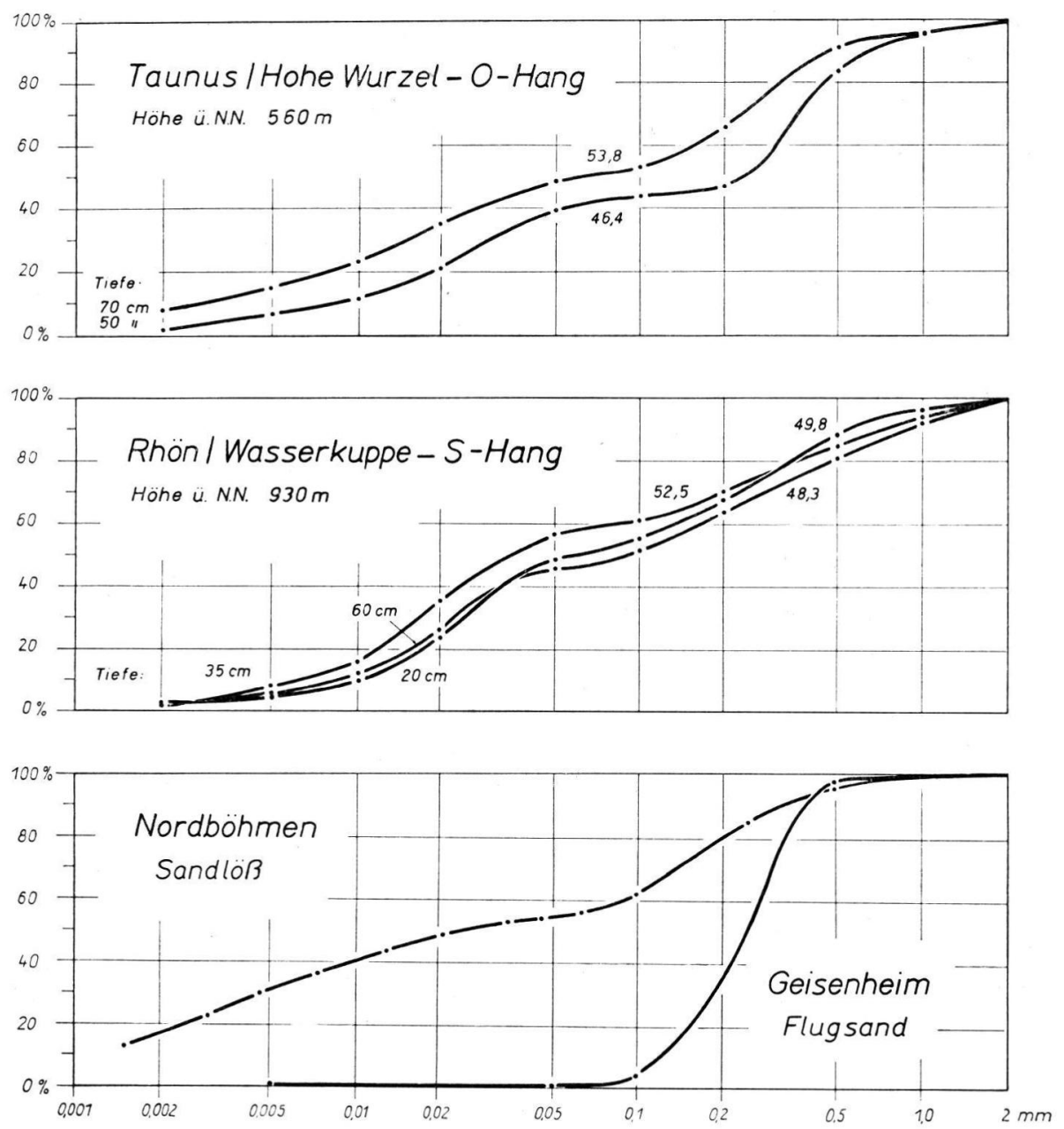

Abb. 4. Kornverteilungskurven der äolischen Deckschicht aus dem Taunus und von der Wasserkuppe. Zum Vergleich sind die Kornverteilungskurven eines Sandlösses aus Böhmen und eines Flugsandes an der Basis des Würm III-Lösses von Geisenheim (Rheingau) angegeben. 
gruppe 0,2-0,5 mm. Gesteine mit Einzelkörnern dieser Größe (Sandsteine) kommen auch in der unmittelbaren Umgebung nicht vor. Es hat daher den Anschein, daß auch an der Wasserkuppe die Lokalfazies eines jungen äolischen Sediments vorliegt. - Nähere Untersuchungen konnten bisher noch nicht durchgeführt werden.

\section{Entstehung und Alter der Deckschichten}

Es erhebt sich nun die Frage: wann und unter welchen Bedingungen sind die Deckschichten entstanden? An ihrer äolischen Entstehung ist wohl nicht zu zweifeln, nachdem durch Körnungsanalysen die für Windsedimente typischen Fraktionen nachgewiesen wurden. Die Ablagerungen können also nur unter einem Klima gebildet worden sein, das zumindest während der eigentlichen Auswehung (Sommer) - relativ trocken gewesen ist. Daß die Deckschichten etwa zur gleichen Zeit wie die würmzeitlichen Lösse entstanden sind, ist wohl so gut wie ausgeschlossen, und zwar aus folgenden Gründen:

1. Der Habitus der Deckschichten ist ein ganz anderer als derjenige des hochglazialen Lösses; äolische Sedimente dieser Art sind aus den Glazial- und Interglazialzeiten nicht bekannt.

2. Würde die Ablagerung im letzten Glazial stattgefunden haben, so wären die lockeren Sedimente wie der Löß längst abgetragen, zumal sie nur geringmächtig sind und sich meist an Stellen finden, die ohne Vegetation einem starken Bodenabtrag unterliegen.

An dieser Stelle soll nun noch die Frage erörtert werden, ob u. U. nicht auch das Wasser an der Bildung der Deckschichten beteiligt gewesen ist, besonders bei den Vorkommen in Hanglage. Zunächst sei hervorgehoben, daß Abschlämmassen von ähnlicher Zusammensetzung niemals das für die Deckschichten charakteristische lockere, hohlraumreiche Gefüge besitzen, selbst dann nicht, wenn Humus in größerer Menge mitverlagert worden ist. Sie sind vielmehr dicht, hohlraumarm und häufig auch plattig, besonders wenn es sich um basenarme Substrate handelt. Abschlämmassen weisen meistens auch in der Vertikalen einen Wechsel der Korngrößenzusammensetzung auf, was auf die durch den Bodenabtrag hervorgerufenen Veränderungen der petrographischen Zusammensetzung des Geländes zurückzuführen ist. So läßt sich z. B. eine zu Beginn der Abtragung vorhanden gewesene Lößdecke durch einen stärkeren Anteil der Staubsandfraktion in den Basisschichten der Abschlämmassen noch erkennen. Mit dem allmählichen Verschwinden der Lößdecke tritt der Staubsand mehr und mehr zugunsten der in dem liegenden Gestein enthaltenen Korngrößen zurück. Solche Veränderungen sind in den äolischen Deckschichten aber nicht nachzuweisen. Gegen die Mitwirkung des Wassers spricht außerdem die fast immer gleichbleibende Mächtigkeit. Wären die Deckschichten durch Wasser entstanden, so müßte eine Abhängigkeit der Mächtigkeit von der Geländeform festzustellen sein. Das ist jedoch nicht der Fall. Die Deckschichten könnten auch nicht die zwischen den Bächen des Oberwaldes liegenden flachen Rücken einnehmen, die bereits während ihrer Ablagerung vorhanden waren. Mehrere Wegeinschnitte zeigten, $\mathrm{da} ß$ die Sedimentation in einem schwachen Relief erfolgte. Diese Beobachtungen und die Korngrößenzusammensetzung lassen daher nur eine äolische Entstehungsweise zu. Dagegen sprechen auch die $\mathrm{ab}$ und $\mathrm{zu}$ vorkommenden Gesteinsstückchen von $1-3 \mathrm{~cm}$ Größe nicht. Sie find fast nur auf die Basiszone der Deckschichten beschränkt und geben daher einen Hinweis darauf, daß auch zu Beginn dieser äolischen Ablagerung zunächst wohl noch das Bodenfließen eine gewisse Rolle spielte. Unter Umständen können die wenigen groben Bestandteile auch durch Auffrieren aus dem liegenden Schutt später nach oben gelangt sein, eine Möglichkeit, auf die mich Herr Prof. Poser in einem brief lich geführten Gedankenaustausch freundlicherweise hinwies. Herr Prof. Poser berichtete in diesem Zusammenhang von ähnlichen Beobachtungen an der Basis äolischer Ablagerungen, wo „Gesteinskomponenten des Untergrundes meistens eine Steil- oder Senk- 
rechtstellung ihrer Längsachsen zeigen, was ein deutlicher Hinweis ist, daß die betreffenden Steine durch frostdynamische Vorgänge erst nach Ablagerung des feinen äolischen Materials wieder in vertikales Wandern kamen".

Die mitgeteilten Beobachtungen führen daher $\mathrm{zu}$ folgendem Ergebnis: Die Deckschichten sind äolisch entstanden und jünger als die Würmvereisung. Als Entstehungszeit kommt nach alledem nur das Spätglazial in Betracht, aus dem ja bereits auch in anderen Gebieten äolische Ablagerungen bekannt sind. $\mathrm{Zu}$ erwähnen sind die Jüngeren Flugdecksande Hollands (T. van Der Hammen 1951, 1952) und Nordwestdeutschlands, sowie die Dünen Nord- und Ostdeutschlands und Polens (H. Poser 1948, 1951). Auch inselartig verbreiteter Löß auf Rügen und die etwas größeren Lößdecken Lettlands seien angeführt (E. SChÖNHALS 1944, 1944a). J. TricarT (1950) berichtet von spätglazialem Löß aus der Umgebung von Straßburg und H. Poser (1951) von Vorkommen aus dem Rhônetal und Nordwestfrankreich. Auch in Ungarn soll nach Untersuchungen von B. Bulla (1938, zitiert nach H. Poser 1951) spätglazialer Löß auf größeren Flächen verbreitet sein.

Für die nähere Bestimmung des Alters der Deckschichten in den hessischen Mittelgebirgen ist der im Vogelsberg an verschiedenen Stellen aufgefundene $\mathrm{Hum}$ u h or i$\mathrm{z}$ o n $\mathrm{t}$ von Bedeutung. Dieser Horizont beweist, daß unmittelbar vor der Anwehung der Deckschicht der Hohe Vogelsberg eine Vegetationsdecke getragen hat, über die mangels Pflanzenresten keine näheren Angaben gemacht werden können. Aller Wahrscheinlichkeit nach handelt es sich um Bodenrelikte des Allerödinterstadials, das ja in Holland deutlich entwickelte terrestrische Humushorizonte hinterlassen hat. Nach den pollenanalytischen Untersuchungen (F. FIRBAS 1949) war Mitteleuropa während des Alleröds bewaldet, und zwar wurden die Wälder nach Nordwesten und Norden birkenreicher und kiefernärmer. Nach der von F. FIRBAS (1949, S. 302) entworfenen Kartenskizze lagen die hessischen Mittelgebirge in dem „Kiefernwaldgebiet mit zurücktretenden Birken". Als vermutlich waldlos wird nur der höchste Teil der Rhön angesehen. Außerhalb Hessens gehören neben den Alpen die höchsten, meist kleinen Teile der Vogesen, des Schwarzwaldes und der Randgebirge Böhmens zur waldlosen Stufe.

Es liegt daher nahe, den im Vogelsberg aufgefundenen Humushorizont der ersten nachweisbaren Waldzeit während des Spätglazials zuzuordnen, wenn wir auch z. Zt. noch nicht in der Lage sind, diese Annahme durch die Pollenanalyse zu bestätigen. Hierzu sind wir aber wohl auch noch aus folgendem Grunde berechtigt: Über dem Humushorizont lagert kein Solifluktionsschutt, auch kein aquatisches Sediment, sondern eine ä ol is che Bildung. Eine solche Abfolge kann aber nur als das Ergebnis eines scharfen Klimawechsels gedeutet werden, durch den die Vegetation stark zurückgedrängt wurde. Dieser Klimarückschlag dürfte am ehesten mit der Jüngeren Tundrenzeit gleichzusetzen sein, die einmal von F. Firbas (1947) als „halbe Eiszeit" bezeichnet wurde. Waldlos wurden nach F. FIRBAS (1949) Gebiete mit einem heutigen Juli-Mittel von $16^{\circ} \mathrm{C}$ und geringer. Subarktische geschlossene Wälder waren in den wärmeren Beckenlandschaften mit einem heutigen Juli-Mittel von $18^{\circ} \mathrm{C}$ und darüber verbreitet, so z. B. im Oberrheintalgraben. Wir können daher für die Hochlagen von Vogelsberg, Taunus und Rhön mit einem heutigen Juli-Mittel von $13-16^{\circ} \mathrm{C}$ eine sehr spärliche Vegetation annehmen. Der Boden wird an zahlreichen Stellen, besonders dort, wo der Wind stark angreifen konnte, kaum Pflanzenwuchs getragen haben. Der oberste humose Horizont und der Mineralboden wurden infolgedessen an zahlreichen Stellen von der äolischen Verlagerung erfaßt, aufbereitet und dorthin transportiert, wo die Kraft des Windes erlahmte oder die Vegetation das Material festhielt.

Abschließend soll noch der Frage nachgegangen werden, ob die Deckschichten Schlüsse auf die Rich tung des Windes zulassen. Änderungen in der Korngrößenzusammensetzung, wie sie im Löß vom Auswehungsgebiet zum Ablagerungsraum fest- 
zustellen sind (E. SchöNHals 1955), können wegen der kleinen Ausdehnung der Decken zur Klärung dieser Frage nicht herangezogen werden. Doch scheinen die Ablagerungen im Taunus gewisse Hinweise auf die Windrichtung zu geben. Fast alle Vorkommen liegen nämlich entweder an SO- oder NO-Hängen. Die größten begleiten die SOHänge, so an der "Platte“ (Abb. 2) und östlich der „Hohen Wurzel“ (Abb. 3). An den W-Hängen findet sich nur ein einziges, äußerst kleines Vorkommen, nämlich am „RoteKreuz-Kopf' (Abb. 3). Die unmittelbar benachbarten 3 liegen am S- bzw. O-Hang. Es besteht also offensichtlich eine deutliche Abhängigkeit der Verbreitung vom Relief, die aller Wahrscheinlichkeit nach auf die Windrichtung zurückzuführen ist. Aus der Lage der Deckschichten darf daher wohl geschlossen werden, daß es sich um Westwinde gehandelt hat, die ja auch von H. Poser $(1950,1951)$ auf Grund anderer Klimazeugen (Dünen) für das Spätglazial Nord- und Westdeutschlands angenommen werden. Weitere gesicherte Angaben über die Windrichtung oder Veränderungen derselben können auf Grund der bisherigen Untersuchungen nicht gemacht werden. Es liegt jedoch die Vermutung nahe, daß die Ablagerungen durch Winde aus verschiedenen Richtungen gebildet worden sind. So könnten die an den SO-Hängen liegenden Decken durch NWbis WNW-Winde, die an den NO-Hängen vorkommenden durch Winde aus südwestlicher Richtung entstanden sein. $\mathrm{Ob}$ diese Windrichtungen zeitlich aufeinander gefolgt sind, kann nicht entschieden werden, da das lehmige, homogene Substrat eine Messung des Einfallwinkels der Schichtung wie bei Dünen oder Sandbänderlössen nicht zuläßt. Solche Messungen führte F. KLUTE (1949) an Dünen zwischen Frankfurt und Darmstadt aus und folgerte, daß die tieferen Schichten bei NNW- bis NW-Winden, die mittleren bei W-Winden und die allerobersten bei WSW-Winden entstanden seien. Eine ähnliche Winddrehung von W auf SW leitete H. Louis (1928) aus Veränderungen der Parabeldünen Norddeutschlands ab. Wie schon betont, reichen die Beobachtungen z. Zt. noch nicht aus, um diese Frage beantworten zu können.

Die beschriebenen äolischen Ablagerungen stellen also wichtige Klimazeugen für die Jüngere Tundrenzeit dar. Das Klima in den Hochlagen der hessischen Mittelgebirge hatte demnach am Ende des Pleistozäns noch einmal für kurze Zeit einen perig la z ia len Charakter, wie dies bereits für den höchsten Teil des Harzes nachgewiesen wurde (H. Poser \& J. Hövermann 1951). Daß die Jüngere Tundrenzeit auch in Nordund Süddeutschland morphologische Zeugnisse hinterlassen hat, haben die Untersuchungen von K. Gripp (1953) an den Seen Ostholsteins und von C. RAthJENs (1953) an den Niederterrassen des Alpenvorlandes gezeigt.

Von großer Bedeutung sind die Ablagerungen auch für die Entwicklungsgeschichte der Böden, insbesondere unserer Lößböden, denn wir haben nun die Möglichkeit, die Böden auf würmzeitlichem Löß mit denen auf den spätglazialen Deckschichten zu vergleichen. Auf diesen Fragenkomplex wird jedoch in einer an anderer Stelle erscheinenden Arbeit näher eingegangen werden.

\section{Schriftenverzeichnis}

Bulla, B.: Der pleistozäne Löß im Karpathenbecken. - Földtani Közlöny 68, S. 33-52, 1938.

Firbas, F.: Uber die späteiszeitlichen Verschiebungen der Waldgrenze. - Naturwiss. 34, S. 114 bis 118, Berlin 1947. - - Spät- und nacheiszeitliche Waldgeschichte Mitteleuropas nördlich der Alpen, Bd. I, 480 S., Jena 1949.

GripP, K.: Die Entstehung der ostholsteinischen Seen und ihre Entwässerung. Beiträge zur Landeskunde von Schleswig-Holstein. - Schr. geogr. Inst. Univ. Kiel, Sonderband, S. 11-26, Kiel 1953.

van der Hammen, T.: Late-glacial flora and periglacial phenomena in the Netherlands. - Leidse Geol. Meded. 17, S. 71-183, 1951 - - Dating and correlation of periglacial deposits in middle en western Europe. - Geologie en Mijnbouw 9, S. 328-336, 1952. 
KLute, F.: Rekonstruktion des Klimas der letzten Eiszeit in Mitteleuropa auf Grund morphologischer und pflanzengeographischer Tatsachen. - Geogr. Rdsch. H. 3 u. 4, S. 81-89 und 121-126, Braunschweig 1949.

Louis, H.: Die Form der norddeutschen Bogendünen. - Z. Geomorphologie 4, 1928/29, S. 7-18, Leipzig 1929.

Rathjens, C.: Uber Klima und Formenschatz der Späteiszeit. - Geologica Bavarica 19, S. 186 bis 194, München 1953.

Poser, H.: Aolische Ablagerungen und Klima des Spätglazials in Mittel- und Westeuropa. Naturwiss. 35, S. 269-276 u. 307-312, Berlin 1948. - - Zur Rekonstruktion der spätglazialen Luftdruckverhältnisse in Mittel- und Westeuropa auf Grund der vorzeitlichen Binnendünen. - Erdkunde 4, S. 81-88, Bonn 1950. - - Die nördliche Lößgrenze in Mitteleuropa und das spätglaziale Klima. - Eiszeitalter und Gegenwart 1, S. 27-55, OOhringen 1951.

Poser, H. \& Hövermann, J.: Untersuchungen zur pleistozänen Harzvergletscherung. - Abh. braunschw. wiss. Ges. 3, S. 62-115, Braunschweig 1951.

Schottlen, W.: Geologische Kaite von Hessen 1:25000, Bl. Ulrichstein mit Erläuterung, 105 S., Darmstadt 1931.

Schönhals, E.: Jungglazialer Löß auf Rügen. - Ber. Reichsamt Bodenforsch., S. 45-49, Wien 1944. - - Spätglazialer Löß in Lettland. - N. Jb. Min. etc. Abt. B, S. 241-250, Stuttgart 1944 (1944a). - - Gesetzmäßigkeiten im Feinaufbau von Talrandlössen mit Bemerkungen über die Entstehung des Lösses. - Eiszeitalter und Gegenwart 3, S. 19-36, Öhringen 1953. - - Kennzahlen für den Feinheitsgrad des Lösses. - Eiszeitalter und Gegenwart 6, S. 133-147, Öhringen 1955.

Soergel, W.: Lösse, Eiszeiten und paläolithische Kulturen. - Jena 1919.

Tricakt, J.: Dépôts würmiens et postwưrmiens du cône de déjection de la Bruche près de Strasbourg. - C. R. somm. Soc. géol. de France, S. 138-139, 1950.

Manuskr. eingeg. 11. 5. 1956.

Anschrift des Verf.: Priv.-Doz. Dr. Ernst Schönhals, Hess. Landesamt für Bodenforschung, Wiesbaden, Mainzer Straße 25.

Zusatz nach Fertigstellung der Arbeit: Im Herbst 1956 fand Verf. im Habichtswald bei Kassel in $500 \mathrm{~m}$ Höhe eine äolische Ablagerung mit den gleichen Eigenschaften wie die hier beschriebenen Vorkommen. Die Beobachtungen werden im Notizblatt des Hessischen Landesamtes für Bodenforschung, 85, Wiesbaden 1957, veröffentlicht. 